\title{
EFEITO DA ADIÇÃO DE PRECURSORES NA PRODUÇÃO DE ALCALOIDE ANTICANCERÍGENO USANDO A TÉCNICA DE PLANEJAMENTO EXPERIMENTAL
}

\author{
Oselys Rodriguez Justo \\ Departamento de Processos Biotecnológicos, Faculdade de Engenharia Química, Universidade Estadual de Campinas, Cidade \\ Universitária "Zeferino Vaz”, CP 6066, 13083-970 Campinas - SP, Brasil \\ Victor Haber-Perez* \\ Setor de Engenharia de Processos, Laboratório de Engenharia de Alimentos, Centro de Ciências e Tecnologias Agropecuárias, \\ Universidade Estadual do Norte Fluminense Darcy Ribeiro, Av. Lamego, 2000, 28013-602 Campos dos Goytacazes - RJ, Brasil \\ Cláudia de Oliveira Viana e Ranulfo Monte Alegre \\ Departamento de Engenharia de Alimentos, Faculdade de Engenharia de Alimentos, Universidade Estadual de Campinas, Cidade \\ Universitária “Zeferino Vaz”, CP 6121, 13083-970 Campinas - SP, Brasil
}

Recebido em 28/4/08; aceito em 19/1/09; publicado na web em 28/7/09

\begin{abstract}
EFFECT OF THE PRECURSOR ADDITION ON THE ANTICANCER ALKALOID PRODUCTION USING EXPERIMENTAL DESIGN METHODOLOGY. The effect of precursors on the anticancer alkaloid production by submerged fermentation using M. anisopliae 3935 was studied, according to complete experimental design $2^{2}$ with three central points. The results showed that lysine was the most important variable, however, when both lysine and glucose were added to the fermentation medium, the alkaloid production reached, approximately, $17 \mathrm{mg} \mathrm{L}^{-1}$ after 120 hours of fermentation. Then, the scale-up of the process was carried out and these results were confirmed. Finally, $35 \mathrm{mg} \mathrm{L}^{-1}$ of alkaloid at $192 \mathrm{~h}$ were attained after increment of added aminoacid lysine.
\end{abstract}

Keywords: alkaloid; fermentation; M. anisopliae.

\section{INTRODUÇÃO}

O composto swainsonine é um alcalóide indolizidínico polihidroxilado $\left(\mathrm{C}_{8} \mathrm{H}_{15} \mathrm{NO}_{3}\right)$ cuja molécula é análoga a açúcares, constituída por anéis piperidínico e pirrolidínico com massa molecular igual 173,2 $\mathrm{g} \mathrm{mol}^{-1}$, cujo nome se deriva de uma planta australiana conhecida como Swainsona canescens. Este composto se apresenta como um promissor agente terapêutico para ser usado na quimioterapia de diversos tipos de neoplasias, devido a sua potencialidade na redução da taxa de crescimento de tumores sólidos e na inibição de metástase de vários tipos de neoplasias. ${ }^{1,2}$ Entre outras propriedades reporta-se também sua capacidade para anular os efeitos citotóxicos de algumas drogas quimioterapêuticas, tais como dexocirubicina e ciclofosfamida, sendo considerado também como um potencial adjuvante terapêutico. ${ }^{3}$

Outras aplicações terapêuticas de alcalóides poli-hidroxilados, incluindo o composto de interesse, estão relacionadas com o tratamento de desordem lisossomal, de processos infecciosos e complicações relacionadas, bem como de doenças autoimunes como artrite e esclerose múltipla. ${ }^{4}$

Particularmente, o princípio terapêutico deste alcalóide baseiase principalmente no seu efeito inibidor de enzimas $\alpha$-manosidase tipo II que participam no processo de glicosilação de proteínas. Estes processos têm sido também associados à presença exagerada de carboidratos na superfície de células cancerígenas e relacionados às doenças progressivas e aos processos de metástase, tendo sido seu efeito amplamente estudado por vários grupos de pesquisa. $^{4-7}$

Inicialmente, este alcalóide foi extraído de sementes de plantas, ${ }^{8}$ posteriormente, obtido a partir de culturas de células vegetais ${ }^{9} \mathrm{e}$, mais recentemente, por fermentação $0^{8,10,11}$ e síntese química. ${ }^{12,13}$ Entretanto, esta última técnica, embora seja a de maior expansão, tem apresen-

*e-mail: victorh@uenf.br tado problemas referentes a seu elevado custo e baixo rendimento na produção, com grande número de operações e etapas de síntese, unido à formação de misturas racêmicas que dificultam a purificação e recuperação do produto final. ${ }^{14} \mathrm{De}$ fato, embora existam algumas propostas para a síntese química assimétrica deste alcalóide, são requeridas ainda múltiplas etapas de reação, prevalecendo os altos custos do processo. ${ }^{15} \mathrm{O}$ processo bioquímico também apresenta problemas operacionais durante a etapa de fermentação, porém, embora ainda pouca atenção tenha sido dada a esta rota, o menor custo deste processo resulta em uma alternativa mais atrativa.

Previamente nosso grupo de pesquisa realizou um screening sobre a capacidade de produção deste alcalóide por culturas de diversas cepas brasileiras de Metarhizium ssp. ${ }^{16}$ e uma linhagem de M. anisopliae 3935 foi a que alcançou, comparativamente entre as cepas avaliadas, maior rendimento de alcalóide produzido (da ordem de $6 \mathrm{mg} \mathrm{L}^{-1}$ ). Neste contexto, este trabalho teve como foco avaliar a potencialidade desta cepa de produzir quantidades maiores de alcalóide em função da adição de precursores, como lisina e glicose, usando a metodologia de planejamento experimental com base nos estudos previamente reportados, ${ }^{10}$ com o propósito de estabelecer uma correlação empírica para estimar a quantidade de alcalóide formado em função dos precursores adicionados em escala de laboratório.

\section{PARTE EXPERIMENTAL}

\section{Micro-organismo e reagentes}

Metarhizium anisopliae 3935 foi obtido da Fundação Tropical de Pesquisa e Tecnologia “André Tosello”, Campinas, Brasil. Alcalóide como padrão analítico, $99 \%$ de pureza, lisina e DL-glicose foram obtidos da Sigma-Aldrich. Aveia em flocos (grau alimentício) foi obtida no mercado nacional. Todos os reagentes utilizados nas análises foram de grau analítico. 


\section{Procedimentos experimentais}

\section{Preparação do inóculo}

Cepa liofilizada de Metarhizium anisopliae 3935, foi reativada em placas de Petri em meio composto por $20 \mathrm{~g} \mathrm{~L}^{-1}$ de aveia comercial e $20 \mathrm{~g} \mathrm{~L}^{-1}$ de agar sendo, então, periodicamente repicada em tubos de ensaios e estocada em geladeira à temperatura de $4{ }^{\circ} \mathrm{C}$. Os inóculos foram preparados em frascos Erlenmayer $(250 \mathrm{~mL})$ no meio descrito anteriormente. Subsequentemente, os esporos formados foram retirados com água destilada e dispersos com agitação em vórtex e adicionados assepticamente nos frascos Erlenmayers ou no biorreator, quando for o caso, para atingir uma concentração inicial de $10^{7}$ esporos $\mathrm{mL}^{-1}$ no processo fermentativo.

\section{Fermentações conduzidas em shaker. Planejamento experimental}

Os ensaios fermentativos foram realizados em frascos Erlenmayer ( $250 \mathrm{~mL}$ ) com $50 \mathrm{~mL}$ de meio estéril a $26^{\circ} \mathrm{C}$ e $200 \mathrm{rpm}$ durante 5 dias. A preparação do meio de cultura foi realizada de acordo com método modificado, reportado por Patrick et al., ${ }^{10}$ usando extrato de aveia comercial $\left(20 \mathrm{~g} \mathrm{~L}^{-1}\right)$, mas cozida até atingir consistência relativa, e suplementado com glicose e aminoácido DL-lisina em várias concentrações. Antes das fermentações, o meio assim preparado foi esterilizado durante 15 min à temperatura de $121^{\circ} \mathrm{C}$ e 15 psi.

No término das fermentações, todos os caldos foram centrifugados (Centrifuga modelo 5804R da Eppendorf) e caracterizados através da determinação de massa seca, $\mathrm{pH}$, concentração de glicose residual e concentração do alcaloide produzido.

Para avaliar os efeitos das concentrações de glicose e de DL-lisina na produção do alcaloide swainsonine, foi realizado um planejamento experimental completo $2^{2}$ com triplicata no ponto central (Tabela 1) para estimativa do erro experimental, durante 5 dias de fermentação conforme as condições de temperatura e agitação descritas anteriormente. Os níveis das variáveis independentes foram definidos em função dos resultados experimentais previamente reportados. ${ }^{10,16} \mathrm{~A}$ análise da significância dos efeitos das variáveis independentes sobre a produção de alcaloide foi avaliada através do programa Statistica, versão 5.0 (StatSoft Co.), enquanto que o programa Design Expert versão 5,0 foi empregado para se realizar a análise da variância (ANOVA) dos resultados obtidos. Neste contexto, o teste F foi empregado como critério de validação da significância estatística dos modelos ajustados.

\section{Fermentações em fase submersa em biorreator tipo tanque agitado}

O meio de cultura foi preparado como descrito anteriormente, porém, constituído por $15 \mathrm{~g} \mathrm{~L}^{-1}$ de extrato de aveia e/ou farelo de arroz, previamente cozido durante $30 \mathrm{~min}$ em água destilada, filtrado e autoclavado durante 15 min à $121^{\circ} \mathrm{C}$ e 15 psi. Como inóculo, uma suspensão de esporos de M. anisopliae obtidos em Erlenmayers com meio inclinado (aproximadamente $100 \mathrm{~mL}$ ) foi transferida assepticamente para o meio de fermentação estéril. A fermentação foi realizada em biorreator tipo tanque agitado New Brunswick Scientific, modelo Bioflo III com capacidade total de $5 \mathrm{~L}$ (aproximadamente $4 \mathrm{~L}$ de volume útil), à $26^{\circ} \mathrm{C}$ e $400 \mathrm{rpm}$. Amostras foram retiradas em intervalos regulares de $24 \mathrm{~h}$ para análise. Durante a fermentação foram adicionadas, de forma intermitente, soluções de $100 \mathrm{mg} \mathrm{L}^{-1}$ de DL-lisina e de $10 \mathrm{~g} \mathrm{~L}^{-1}$ de glicose, respectivamente.

\section{Métodos analíticos}

\section{Determinação de massa seca}

Para a determinação da biomassa celular resultante das culturas em Erlenmayers, as amostras foram filtradas, então, as células separadas foram lavadas duas vezes com água destilada e posteriormente, as mesmas foram secas até peso constante, à temperatura de $65^{\circ} \mathrm{C} \mathrm{e}$ $8 \mathrm{kPa}$, usando estufa a vácuo modelo GST-920 (Suprilab, Brasil).

No caso dos experimentos conduzidos em biorreator, a determinação de biomassa foi realizada por análise turbidimétrica das amostras de caldo de fermentação tomadas a intervalos regulares de $24 \mathrm{~h}$, de acordo com medições de densidade ótica a $660 \mathrm{~nm}$ usando um espectrofotômetro Hach DR/4000V.

\section{Determinação de $\mathrm{pH}$}

$\mathrm{O}$ pH foi determinado para cada uma das amostras por leitura direta em equipamento DIGIMED, modelo DM 20.

\section{Análise da produção de alcaloide}

A concentração de alcaloide produzido foi determinada por ESI-MS em modo positivo usando espectrômetro de massas Q-TOF (Micromass, Manchester, UK) de acordo com as condições previamente estabelecidas. ${ }^{16}$ Para a quantificação do alcaloide uma curva padrão foi preparada adicionando-se $0,5 \mu \mathrm{L}$ de ácido fórmico e uma solução de metanol (1:1 v/v) à solução de alcaloide padrão $(500 \mu \mathrm{L})$. As amostras de fermentação também foram preparadas como descrito acima, cujas análises foram feitas por infusão direta com um fluxo de

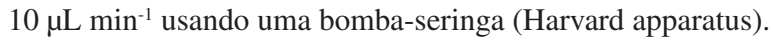

\section{RESULTADOS E DISCUSSÃO}

Na Tabela 1 são apresentados os resultados do planejamento experimental realizado em shaker e conforme pode ser observado, as concentrações do alcaloide produzido variaram de 2,2 a 16,8 $\mathrm{mg} \mathrm{L}^{-1}$, sendo este último valor praticamente 3 vezes superior ao alcançado em trabalhos anteriores ${ }^{16}$ com a mesma cepa. Neste caso, o melhor resultado (ensaio 4) foi obtido quando a cultura foi conduzida em meio com as maiores concentrações de lisina $\left(170 \mathrm{mg} \mathrm{L}^{-1}\right)$ e glicose $\left(10 \mathrm{~g} \mathrm{~L}^{-1}\right)$, durante 5 dias de fermentação.

Tabela 1. Matriz experimental e resultados do planejamento estatístico para a produção de alcaloide por fermentação durante 5 dias usando M. anisopliae 3935 à temperatura de $26^{\circ} \mathrm{C}$

\begin{tabular}{|c|c|c|c|c|c|c|}
\hline Ensaio & $\mathrm{X}_{1}$ & $\mathrm{X}_{2}$ & DL-lisina $\left(\mathrm{mg} \mathrm{L}^{-1}\right)$ & Glicose $\left(\mathrm{g} \mathrm{L}^{-1}\right)$ & Massa Seca $\left(\mathrm{g} \mathrm{L}^{-1}\right)$ & Alcaloide $\left(\mathrm{mg} \mathrm{L}^{-1}\right)$ \\
\hline 1 & -1 & -1 & 30 & 5,0 & 5,998 & 2,2 \\
\hline 2 & +1 & -1 & 170 & 5,0 & 5,636 & 9,8 \\
\hline 3 & -1 & +1 & 30 & 10,0 & 6,450 & 9,4 \\
\hline 4 & +1 & +1 & 170 & 10,0 & 6,268 & 16,8 \\
\hline 5 & 0 & 0 & 100 & 7,5 & 6,166 & 7,7 \\
\hline 6 & 0 & 0 & 100 & 7,5 & 6,308 & 5,6 \\
\hline 7 & 0 & 0 & 100 & 7,5 & 5,760 & 8,2 \\
\hline
\end{tabular}


De acordo com o gráfico de Pareto (Figura 1), resultante do processamento estatístico dos dados experimentais (Tabela 1) usando o software Statistica v. 7.0, a análise da significância dos efeitos das variáveis independentes estudadas revelou que ambas foram significativas ao nível de $95 \%$ de confiança, enquanto que a interação entre elas só se mostrou significativa em um intervalo de confiança menor (90\%). Analisando-se o efeito de cada variável independente sobre a resposta, observou-se que aquela que teve maior efeito foi a quantidade de lisina adicionada ao meio de cultura, seguida da concentração de glicose. Então, um aumento da concentração destas duas variáveis provavelmente levaria à obtenção de maiores valores de concentração de alcalóide.

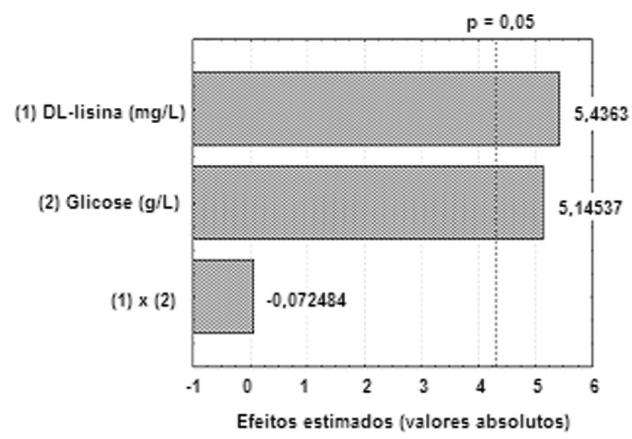

Figura 1. Efeitos das variáveis independentes estudadas e de sua interação sobre a concentração de swainsonine, produzido durante 5 dias de fermentação, para um nível de confiança de $95 \%(p<0,05)$

Do ponto de vista comparativo, os resultados obtidos neste trabalho foram aproximadamente 91 e $24 \%$ maiores que os reportados por Hino et al. ${ }^{17}$ e Patrick et al., ${ }^{9}$ respectivamente, em termos de produtividade e tomando como base a quantidade de alcaloide produzido durante 170 h de fermentação. Por outro lado, estes últimos autores, os quais já tinham discutido a importância da adição de isômeros do aminoácido lisina (L-lisina, D-lisina e DL-lisina) na produção deste alcaloide, alcançaram valores acima de $30 \mathrm{mg} \mathrm{L}^{-1}$ de alcaloide em 190 h, porém, usando uma linhagem diferente de Metarhizium sp. e $2 \%$ de aminoácidos no meio de cultura. No entanto, estes autores não estabeleceram uma correlação entre os diferentes tipos de substrato no que se refere à fonte de carbono e ao uso de isômeros do aminoácido lisina usados como suplemento do meio de cultura e a produção de alcaloide, mas, os melhores resultados foram observados quando se utilizou o meio que continha extrato de aveia e D-lisina. Entretanto, o fato da D-lisina apresentar um custo bem mais elevado que a DLlisina constitui um problema prático para sua implementação.

Ao mesmo tempo, estes autores estudaram a influência de oxigênio dissolvido na morfologia do fungo empregado e na produção de alcalóide, cujos resultados mostraram que altas concentrações de oxigênio dissolvido resultaram na formação de aglomerados de fungos e em concentrações de alcalóide de $1,56 \mathrm{mg} \mathrm{L}^{-1}$, depois de $220 \mathrm{~h}$ de fermentação. Por outro lado, em baixas concentrações, observou-se a manutenção do crescimento hifal com $6,41 \mathrm{mg} \mathrm{L}^{-1} \mathrm{de}$ alcaloide para intervalos de tempo similares ao anterior. Assim, os autores concluíram que a oxigenação excessiva levou à redução no rendimento do processo, de modo que, apesar de ser necessária alta aeração para o crescimento do fungo, isto não era essencial para a produção de alcaloide. Depois de 250 h de fermentação, as curvas de crescimento declinaram.

Neste trabalho, de acordo com o resultado da significância dos efeitos e considerando o critério de hierarquia, um modelo empírico (Equação 1) em função dos termos codificados foi ajustado para predizer a concentração de alcaloide $\left(\mathrm{C}_{\text {alc }}\right)$ em função das concentrações dos precursores no meio de cultura.

$C_{\text {alc. }}=9,55+3.75 X_{1}+3,55 X_{2}-0,05 X_{1} X_{2}$

A Tabela 2 apresenta os resultados da análise da variância (ANOVA) ao nível de confiança de $90 \%$ ( $\mathrm{p}<0.10)$. O valor do coeficiente de correlação $\left(\mathrm{R}^{2}=0,965\right)$ obtido para esta análise revelou a qualidade do ajuste deste modelo matemático. Fato que também foi confirmado pela análise do teste $F$, uma vez que o valor de $\mathrm{p}(0,051)$ foi muito menor que 0,1000 . Segundo Barros et al. ${ }^{18}$ se verifica que, se o F calculado for maior que o tabelado para o nível de confiança adotado, há evidência estatística suficiente para supor a existência de uma relação entre as variáveis. No entanto, notamos que isso não implica necessariamente que a relação encontrada seja um modelo apropriado para prever a concentração de alcaloide como uma função da concentração de precursores, lisina e glicose. Assim sendo, se desconsideramos o termo da interação (Equação 1) a existência de curvatura sugere que mais testes de adequação do modelo serão requeridos antes de ficarmos confortáveis em usar esta equação para realizar previsões técnicas, mesmo não havendo sido encontrada du-

Tabela 2. Análise de variância (ANOVA) usando o programa Design expert para a variável resposta (concentração de alcalóide produzido), ao nível de confiança de $90 \%$

\begin{tabular}{|c|c|c|c|c|c|}
\hline Fonte da variação & Soma quadrática & Graus de Liberdade & Média Quadrática & $\mathrm{F}_{\text {cal }}$ & $\mathrm{p}<0,100$ \\
\hline Modelo & 106,67 & 3 & 35,56 & 18,68 & 0,0512 \\
\hline $\mathrm{x}_{1}$ & 56,25 & 1 & 56,25 & 29,55 & 0,032 \\
\hline $\mathrm{x}_{2}$ & 50,41 & 1 & 50,41 & 26,49 & 0,0357 \\
\hline $\mathrm{x}_{1} \mathrm{x}_{2}$ & $1 \times 10^{-2}$ & 1 & $1 \times 10^{-2}$ & $5,25 \times 10^{-3}$ & 0,9488 \\
\hline Curvatura & 9,74 & 1 & 9,74 & 5,12 & 0,1521 \\
\hline Erro puro & 3,81 & 2 & 1,90 & & \\
\hline Total & 120,21 & 6 & & & \\
\hline
\end{tabular}

Valores de p menores que 0,1 indicam que os termos do modelo são significativos. 
rante a análise falta de ajuste. Neste contexto, adotamos o critério de "hierarquia" para garantir um melhor ajuste do modelo, preservando o termo da interação na Equação 1 e garantindo que a curvatura não seja estatisticamente significante.

Na prática, pode-se considerar uma regressão como útil para fins de previsão se o valor do $\mathrm{F}$ calculado for, pelo menos, de 4 a 10 vezes maior que o valor do ponto de distribuição $\mathrm{F}$ com o número apropriado de graus de liberdade, ao nível de confiança escolhido. ${ }^{19}$

O gráfico de superfície de resposta (Figura 2) mostrou que para o nível de confiança escolhido (90\%) a interação entre as duas variáveis independentes teve efeito positivo, indicando que para otimizar a concentração do alcaloide produzido durante 5 dias de fermentação seria conveniente aumentar as concentrações de lisina e glicose no meio de cultura. Contudo, nestas novas condições seria necessário realizar um estudo para saber se o sistema experimental não seria limitado pelos custos envolvidos com estes compostos.

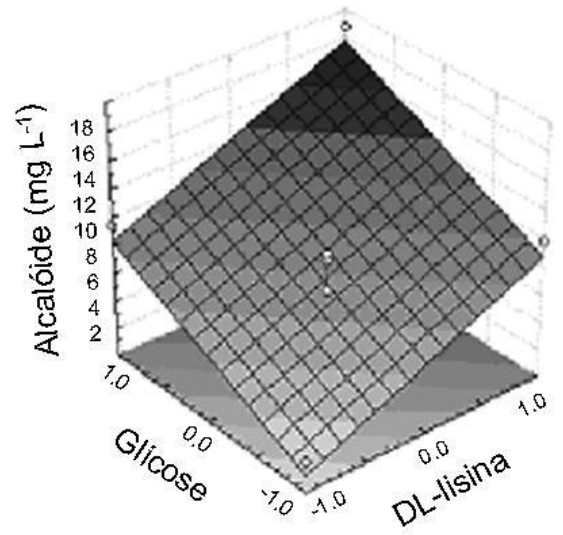

Figura 2. Gráfico tridimensional da superficie de resposta para o planejamento experimental realizado (nível de confiança de 90\%) mostrando o efeito da interação entre as variáveis: concentração inicial de DL-Lisina e de glicose na concentração do alcaloide swainsonine, produzido durante 5 dias de fermentação

\section{Fermentação em biorreator}

Com base nos resultados do planejamento estatístico, visando a ampliação de escala e a validação dos resultados encontrados, realizou-se um experimento de fermentação em biorreator tipo tanque agitado com a melhor condição encontrada. Além disso, foram efetuadas duas adições intermitentes de glicose e DL-lisina, uma vez que se mostraram como variáveis importantes para uma maior produção do alcalóide.

Inicialmente, os meios de fermentação apresentaram cor branca devido à presença intacta do amido. Nas primeiras $50 \mathrm{~h}$ de fermentação o consumo de glicose manteve-se praticamente constante, sugerindo-nos que ainda não havia biomassa suficiente para degradar o substrato. No decorrer do processo, o caldo de fermentação foi se tornando cada vez mais turvo, sugerindo o consumo dos nutrientes pelo fungo. Nesta etapa, houve problemas na tomada de amostras representativas do caldo de fermentação; o comportamento reológico do caldo apresentou alterações em decorrência da migração de hifas e esporos para a parte superior das paredes do fermentador. Assim, houve a formação de uma espessa torta de biomassa na superfície do líquido (Figura 1S - Material Suplementar), resultando, consequentemente, em problemas de transferência de massa e na determinação de biomassa celular no seio do caldo de fermentação. Resultados similares, com relação a este tipo de problema reológico durante a cultura de linhagens diferentes de M. anisopliae, foram também reportados. ${ }^{20,21}$ Similarmente, durante as culturas em shaker foi observado este comportamento, isto é, formou-se uma torta na superfície do caldo de cultura dentro dos frascos Erlenmayer, contudo, o problema foi menos complicado para a determinação da biomassa porque, neste caso, todo meio foi filtrado e a torta resultante usada para análise.

Os perfis de $\mathrm{pH}$, concentração de glicose e concentração de swainsonine obtidos para esta fermentação são apresentados na Figura 3. Durante este experimento o $\mathrm{pH}$ não foi controlado para evitar retardo no crescimento do fungo. ${ }^{11,22} \mathrm{O}$ valor de $\mathrm{pH}$ inicial da cultura, que era de 6,4 caiu eventualmente, havendo uma estabilização em torno de 3,5 . Esta queda coincidiu com o aumento da produção de alcaloide. Resultados similares foram observados por Tamerler et al., ${ }^{11}$ os quais sugeriram que essa queda no $\mathrm{pH}$ do meio poderia ser explicada pela secreção de ácidos orgânicos durante o período de crescimento rápido do fungo, sendo que o controle do $\mathrm{pH}$ durante a fermentação não foi favorável à produção do alcaloide.

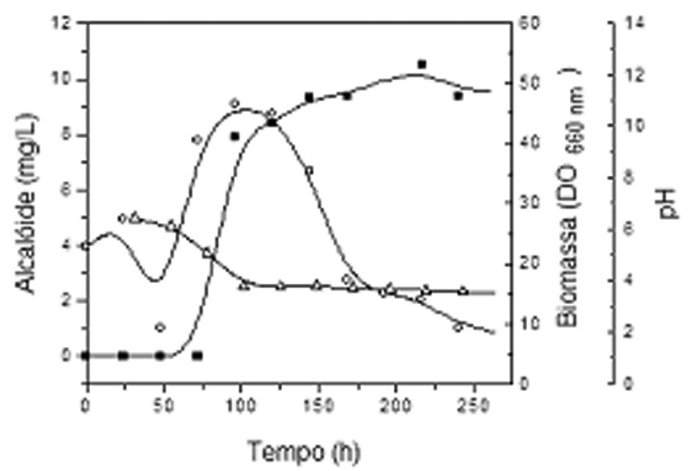

Figura 3. Resultados da produção de alcalóide por M. anisopliae $3935 \mathrm{em}$ biorreator tipo tanque agitado, realizada à temperatura de $26^{\circ} \mathrm{C}, 0,6 \mathrm{vvm}$ e $400 \mathrm{rpm}$. - Concentração de alcaloide (Swainsonine) em $\mathrm{mg} \mathrm{L}^{-1}$; $\bigcirc$ Crescimento celular (Densidade ótica a $660 \mathrm{~nm}$ ) e $\Delta$ - pH

Verificou-se também que, após a adição intermitente de glicose e DLlisina ao meio de fermentação às 144 h, houve incremento na produção de alcalóide. Neste contexto, os espectros de massas revelaram que as concentrações de alcaloide foram maiores que $11,5 \mathrm{mg} \mathrm{L}^{-1}$. Assim, o perfil da curva de produto mostra que a produção do alcaloide se inicia a partir das primeiras $96 \mathrm{~h}$ de fermentação, tendo um acréscimo até atingir seu valor máximo em uma concentração de aproximadamente $11 \mathrm{mg} \mathrm{L}^{-1}$ às $216 \mathrm{~h}$, mantendo-se praticamente constante até $240 \mathrm{~h}$ de fermentação, momento no qual o processo foi interrompido. Nota-se ainda que, ao comparar estes resultados com aqueles obtidos em frascos Erlenmayers, há uma relativa diferença na concentração de alcaloide produzido e que em termos de produtividade os valores de alcaloide produzidos apenas nas primeiras $120 \mathrm{~h}$ de fermentação foram comparativamente superiores aos reportados por Sim e Perry. ${ }^{22}$ Este comportamento evidencia o efeito dos problemas decorrentes das características do biorreator, bem como das condições operacionais estabelecidas. Certamente, estes resultados podem ter sido influenciados pela migração das células para a superfície do caldo de fermentação, como ressaltado anteriormente, resultando em uma apreciável carência de biomassa no meio, o que foi corroborado pelo perfil da curva de crescimento celular que após 100 h de fermentação, aproximadamente, apresentou um decréscimo na biomassa celular.

Simultaneamente, o desempenho da fermentação também foi acompanhado através de análises microscópicas (Figura 2S), feitas nas amostras retiradas do caldo de fermentação em diferentes intervalos de tempo, com o propósito de verificar alterações morfológicas do fungo durante o processo. Conforme se pode observar, nas primeiras horas de fermentação não houve formação de hifas, verificando-se predominantemente a presença de esporos (Figura 
2Sa). Posteriormente, começou o crescimento do fungo e observou-se uma visível fragmentação das hifas, provavelmente devido ao estresse induzido pelas pás do impulsor, causando uma diferença de esporos no seio do líquido (Figura 2Sb). Entretanto, esta suspensão celular apresentou um maior número de hifas em relação ao primeiro caso, possivelmente como resultado da adição de precursores levando a um aumento de biomassa. Esta fragmentação das hifas se manteve durante as próximas horas de cultura (Figura $2 \mathrm{Sc}$ e $2 \mathrm{Sd}$ ), havendo ao mesmo tempo uma mudança morfológica gradativa das hifas livres para emaranhadas, porém, não foi observada a formação de pellets. Já na Figura 2Sd, observou-se suspensão com maior número de hifas na amostra que em casos anteriores, provavelmente por causa da adição de lisina que pode ter estimulado um aumento de biomassa, mas, já migrada para a superfície do caldo de fermentação.

A partir das dificuldades encontradas, decidiu-se aumentar as concentrações de DL-lisina no meio de fermentação. Os experimentos foram conduzidos em duplicata por 8 dias nas mesmas condições de agitação e temperatura, porém variando-se a concentração de DL-lisina de 0 a $4 \mathrm{~g} \mathrm{~L}^{-1}$. Os resultados (Figura 4) foram animadores desde que concentrações médias de $34,5 \mathrm{mg} \mathrm{L}^{-1}$ de alcaloide foram alcançadas quando $1 \mathrm{~g} \mathrm{~L}^{-1}$ de aminoácido foi usado como precursor. Entretanto, concentrações maiores de aminoácido resultaram em inibição da produção extracelular de alcaloide, embora neste trabalho não tenham sido avaliadas as concentrações intracelulares deste produto. As razões para este comportamento não são claras, mas a princípio os resultados sugerem uma possível existência de inibição feedback, uma vez que o catabolismo do aminoácido L-lisina acontece via uma sequência de passos de ruptura oxidativa pela enzima saccharopine oxidase e posteriores passos de redução para resultar em ácido pipecólico, ${ }^{23}$ que é o precursor do alcaloide de interesse ou talvez pela inibição resultante da ação tóxica da forma D do aminoácido que, agora em maiores concentrações, fez com que o microorganismo respondesse de maneira diferente.

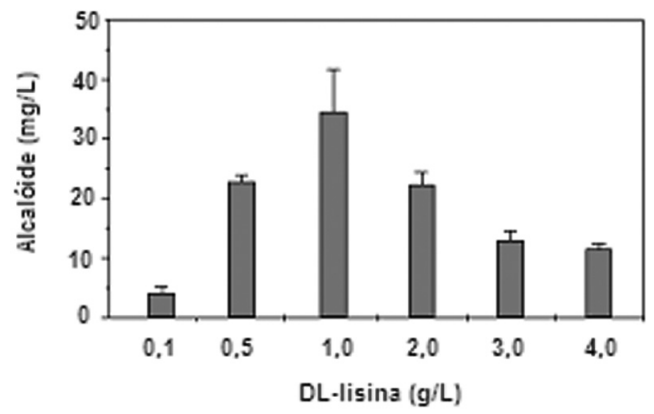

Figura 4. Efeito da concentração de DL-lisina na produção de alcaloide durante a fermentação de M. anisopliae 3935 à temperatura de $26^{\circ} \mathrm{Ce} 200$ rpm durante 8 dias de fermentação

\section{CONCLUSÕES}

A produção de alcaloide anticancerígeno por uma linhagem de $\mathrm{M}$. anisopliae 3935 foi estudada. O efeito da adição de precursores foi verificado e um modelo matemático para estimar este comportamento foi ajustado. Os melhores resultados foram alcançados nas fermentações realizadas durante 8 dias, onde os valores máximos de alcaloide produzido foram observados quando as mais altas concentrações de precursores foram usadas. No entanto, os resultados sugerem que em processos mais longos a concentração do alcaloide produzido não depende apenas das concentrações iniciais dos precursores e, portanto, adições intermitentes são requeridas ao longo do processo. Por outro lado, os problemas reológicos que conduziram a limitações de transferência de massa durante as fermentações sugerem o estudo da obtenção deste alcalóide por fermentação sobre substratos sólidos como uma alternativa mais promissora, a qual deverá ser avaliada em trabalhos posteriores.

\section{MATERIAL SUPLEMENTAR}

No material suplementar, disponível no endereço eletrônico http:// quimicanova.sbq.org.br, na forma de arquivo PDF, encontram-se as Figuras $1 \mathrm{~S}$ e $2 \mathrm{~S}$.

\section{AGRADECIMENTOS}

Ao apoio financeiro da FAPESP e do CNPq, bem como ao Prof. Dr. M. N. Eberlin e ao Dr. R. R. Catharino pelo auxílio na determinação de alcaloide usando a técnica de ESI-MS no Laboratório Thomson de Espectrometria de Massas do Instituto de Química da UNICAMP.

\section{REFERÊNCIAS}

1. Sun, J. Y.; Zhu, M. Z.; Wang, S. W.; Miao, S.; Xie, Y. H.; Wang, J. B.; Phytomedicine 2007, 14, 353 .

2. Lagana, A.; Goetz, J. G.; Cheung, P.; Raz, A.; Dennis, J. W.; Nabi, I. R.; Mol. Cell. Biol. 2006, 26, 3181.

3. Oredipe, O. A.; Furbert-Harris, P. M.; Laniyan, I.; Green, W. R.; Griffin, W. M.; Sridhar, R.; Cell. Mol. Biol. 2003, 49, 1089.

4. Watson, A. A.; Fleet, G. W. J.; Asano, N.; Molyneux, R. J.; Nash, R. J.; Phytochemistry 2001, 56, 265.

5. Misago, M.; Tsukada, J.; Fukuda, M. N.; Eto, S.; Biochem. Biophys. Res. Commun. 2000, 269, 219.

6. Oredipe, O. A.; Furbert-Harris, P. M.; Laniyan, I.; Green, W. R.; White, S. L.; Olden, K.; Parish-Gause, D.; Vaughn, T.; Griffin, W. M.; Sridhar, R.; Int. Immunopharmacol. 2003a, 3, 445.

7. Oredipe, O. A.; Furbert-Harris, P. M.; Laniyan, I.; Griffin, W. M.; Sridhar, R.; Int. Immunopharmacol. 2003b, 3, 1537.

8. Dorling, P. R.; Colegate, S. M.; Huxtable, C. R. Em Swainsonine and related glycosidase inhibitors; James, L. F.; Elbein, A. D.; Molyneux, R. J.; Warren, C. D., eds.; Iowa State University Press: Iowa, 1989.

9. Gardner, D. R.; Molyneux, R. J.; Ralphs, M. H.; J. Agric. Food Chem. 2001, 49, 4573 .

10. Patrick, M. S.; Adlar, M. W.; Keshavarz, T.; Biotechnol. Lett. 1993, 15, 997.

11. Tamerler, C. Y.; Ullah, M.; Adlar, M. W.; Keshavarz, T.; FEMS Microbiol. Lett. 1998, 168, 17.

12. Nemr, A. E.; Tetrahedron 2000, 56, 8579.

13. Yuan, X.; Linhardt, R. J.; Curr. Top. Med. Chem. 2005, 5, 1393.

14. Pyne, S. G.; Curr. Org. Synth. 2005, 2, 39.

15. Ceccon, J.; Greene, A. E.; Poisson, J.; Org. Lett. 2006, 8, 4739.

16. Justo, O. R.; Calheiros, C. S.; Perez, V. H.; Alegre, R. M.; Braz. J. Microbiol. 2007, 38, 780.

17. Hino, M.; Nakayama, O.; Tsurimi, Y.; Adachi, K.; Shibata, T.; Terano, H.; Kohsaka, M.; Aoki, H.; Imanaka, H. ; J. Antibiotics 1985, 38, 926.

18. Barros Neto, B.; Scarminio, I. S.; Bruns, R. E.; Como fazer experimentos: Pesquisa e desenvolvimento na ciência e na industria, Editora da Unicamp: Campinas, 2001.

19. Box, G. E.; Draper, N. R.; Empirical model-building and response surfaces, John Wiley: New York, 1987.

20. Patrick, M. S.; Adlar, M. W.; Keshavarz, T.; Enzyme Microb. Tech. 1996, $18,428$.

21. Keshavarz, T.; Adlard, M. A.; The First European Congress on Chemical Engineering, Proceedings 1997, 4, 2543.

22. Sim, K. L.; Perry, D.; Mycol. Res. 1995, 99, 1078.

23. He, M. J.; Ind. Microbiol. Biotechnol. 2006, 36, 401. 


\section{EFEITO DA ADIÇÃO DE PRECURSORES NA PRODUÇÃO DE ALCALOIDE ANTICANCERÍGENO USANDO A TÉCNICA DE PLANEJAMENTO EXPERIMENTAL}

\section{Oselys Rodriguez Justo}

Departamento de Processos Biotecnológicos, Faculdade de Engenharia Química, Universidade Estadual de Campinas, Cidade Universitária “Zeferino Vaz”, CP 6066, 13083-970 Campinas - SP, Brasil

\section{Victor Haber-Perez*}

Setor de Engenharia de Processos, Laboratório de Engenharia de Alimentos, Centro de Ciências e Tecnologias Agropecuárias, Universidade Estadual do Norte Fluminense Darcy Ribeiro, Av. Lamego, 2000, 28013-602 Campos dos Goytacazes - RJ, Brasil Cláudia de Oliveira Viana e Ranulfo Monte Alegre

Departamento de Engenharia de Alimentos, Faculdade de Engenharia de Alimentos, Universidade Estadual de Campinas, Cidade Universitária “Zeferino Vaz”, CP 6121, 13083-970 Campinas - SP, Brasil

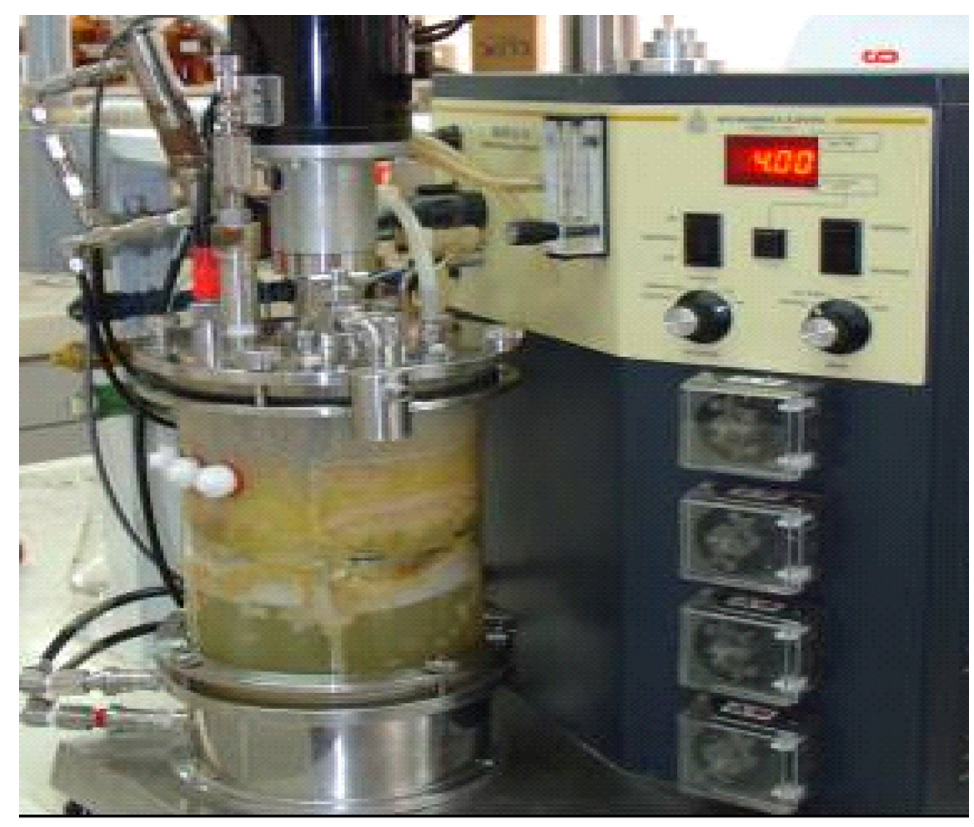

Figura 1S. Ilustração da formação de uma torta como resultado da migração dos micro-organismos do seio do caldo de fermentação para a superfície durante a fermentação submersa em biorreator tipo tanque agitado à temperatura de $26{ }^{\circ} \mathrm{Ce} 400 \mathrm{rpm}$ e $0,6 \mathrm{vvm}$, de M. anisopliae 3935 
a)

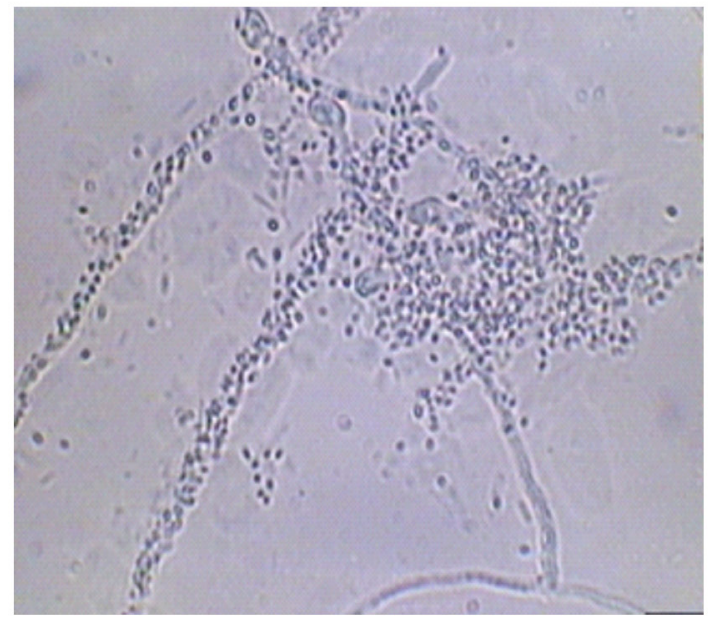

c)

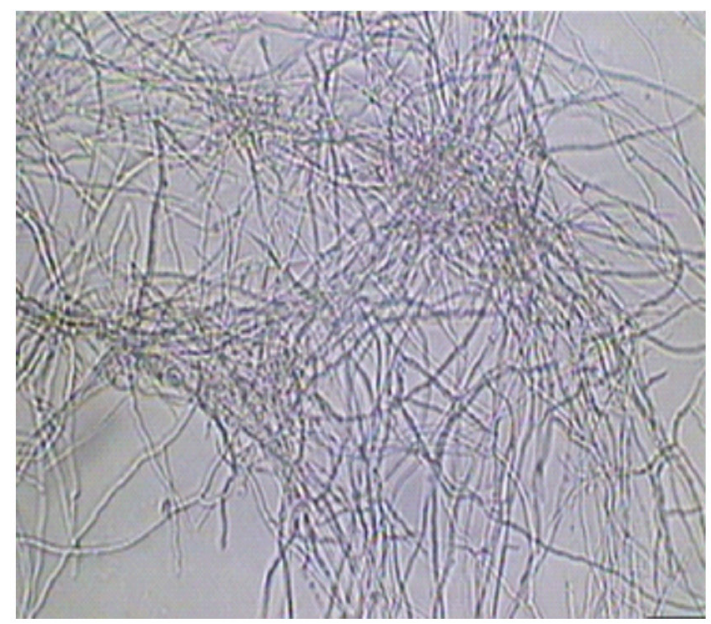

b)

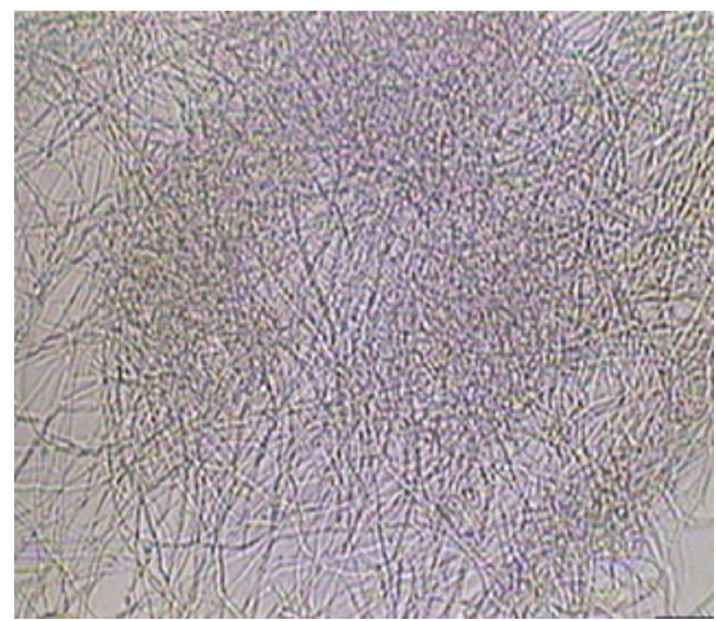

d)

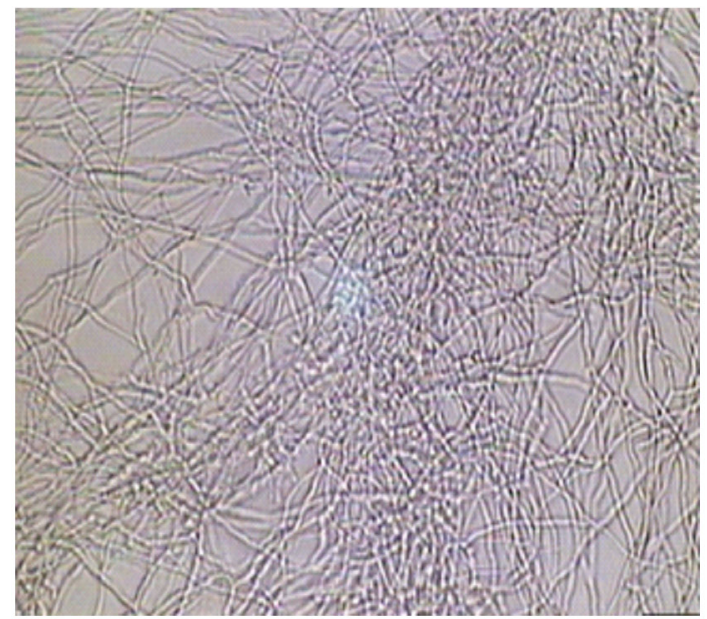

Figura 2S. Microscopia (aumento de 400 vezes) de amostras de fermentação de M. anisopliae em biorreator tipo tanque agitado, tomadas a diferentes tempos de fermentação: a) $72 \mathrm{~h}$; b) $172 \mathrm{~h}$; c) $192 \mathrm{~h} \mathrm{e} \mathrm{d)} 240 \mathrm{~h}$ 